\title{
Philosophiques
}

\section{Jeffrey Andrew Barash, Heidegger et son siècle, Paris, PUF (coll. " Pratiques théoriques »), 1995, 188 p.}

\section{Yvon Corbeil}

Volume 25, numéro 2, automne 1998

Les modèles d'évolution en économie et en sciences sociales

URI : https://id.erudit.org/iderudit/027494ar

DOI : https://doi.org/10.7202/027494ar

Aller au sommaire du numéro

Éditeur(s)

Société de philosophie du Québec

ISSN

0316-2923 (imprimé)

1492-1391 (numérique)

Découvrir la revue

Citer ce compte rendu

Corbeil, Y. (1998). Compte rendu de [Jeffrey Andrew Barash, Heidegger et son siècle, Paris, PUF (coll. " Pratiques théoriques »), 1995, 188 p.] Philosophiques, 25(2), 288-293. https://doi.org/10.7202/027494ar d'utilisation que vous pouvez consulter en ligne.

https://apropos.erudit.org/fr/usagers/politique-dutilisation/ 
Jeffrey Andrew Barash, Heidegger et son siècle, Paris, PUF (coll. " Pratiques théoriques »), 1995, $188 \mathrm{p}$.

Le récent ouvrage de Jeffrey Andrew Barash, professeur à l'Université de Picardie (Amiens), est un recueil d'articles parus entre 1982 et 1993. Son unité n'en est pas moins évidente, qui tient à l'intérêt principal de l'auteur pour la contextualisation historique de l'œuvre de Heidegger, ainsi qu'à la méthode interprétative qu'il met en ceuvre pour celle-là, et qui se veut à contre-courant de certaines postérités heideggeriennes, notamment celles qu'assument la phénoménologie française ou la pensée de 
la * fin de la métaphysique . Les thèmes qui intéressent plus particulièrement Barash concernent essentiellement l'histoire et gravitent autour de la conception qu'en propose Heidegger, explicitement ou non. L'auteur s'intéresse également aux problèmes reliés à la Kehre et à ses implications pour la conception de l'histoire qui affleure depuis l'œuvre heideggerienne, ainsi qu'à l'intégration de cette dernière dans son propre contexte historique. La méthode qu'il emploie - commune à tous les articles réunis dans ce recueil et similaire à celle qui sous-tend son ouvrage de 1988, Martin Heidegger and the Problem of Historical Meaning -- s'inscrit en faux contre une lecture immanentiste de l'ceuvre et refuse ainsi d'emblée les règles du jeu interprétatif qu'énonce fréquemment Heidegger, et qu'il espère voir appliquer à ses propres travaux. Barash commente sa propre méthode en écrivant qu'elle vise à "écarter un mode de compréhension qui a voulu s'enfermer dans les limites de la méthode heideggerienne elle-mêrne " (p. 10). C'est dire qu'il refuse la prétention heideggerienne selon laquelle il faut lire son oxuve, et après lui toute aeuvre philosophique produite arant lui, aux lumières que procure la eerljtude d'une fin de la métaphysique, ou à celles oetroyées par le fait de savoir que toute la philosophie oceidentale est marquée du recouvrenent antique de la vérité de l'être, recouvrement dont cette philosophie ne ferait que relaler les péripélies. Mais l'élection, à titre de perspective majeure visant l'interprétation de toute l'ouvre heideggerienne, d'un thème qui ne constitue pas la principale préoccupation de celle-ci et qui, de surcroît, reçoit, selon les ouvrages et les époques envisagés, des caractérisations qui défient toute interprétation unifiante ne va pas sans difficulté. Naviguant dans ces eaux traîtresses de la pensée de Heidegger qui affirment sinultanément le nécessaire caractère historique de toute pensée et la secondarité de cette historicité eu égard aux a problèmes fondamentaux " des " essences " qu'elle met en ceuvre et en face desquelles toute contextualisation apparaît secondaire, Barash garde tant bien que mal le cap sur une interprétation unifiante qui voudrait éclairer l'o:uvre heideggerienne à la lumière d'une unique préconception de l'histoire. Mais cette ceuvre, déjà complexe dans sa première phase (disons jusqu'à 1929) quant à sa pensée de l'histoire, le devient encore plus par la suite, dès lors que survienment les bouleversements vecasiommés par la kehre et qui tendent à recontextualiser - mais d'une certaine manjère - ce qui, d'abord, caractérisait toute l'histoire et, done, pouvait apparaître comme tout à fait anhistorique.

L'u histoire ", selon lleidegger, est celle de la pensée occidentale qui, de Platon aux Temps Modernes, narre les péripéties de l'auto-déploiement de la pensée rationnelle à partir d'un recouvrement initial, fautif certes, nais qui n'en est pas moins essenticl, dans la mesure où toute l'histoire est à comprendre à partir cle celui-ci et où tout au-delà de ce recouvrement est quant à lui incompréhensible (à tout le noins par l'entrenise d'une pensée qui n'est que la résultante de ce recouvrenent el qui, clès lors, est à ce point déterminée par celui-ci qu'elle ne peut mettre à jour que ce cu'il lui suggère). La confusion est toujours possible entre cette enception de l' histoire met le caractère fondamentalement historial que Hejdegger attribue au Dasein. Celte demière earactéristique constitutive préside en effet notamment à l' analytique existentiale " que conduit Sein und Zeil $\mid S Z$ el vise plus particulièrement à contrer une certaine pensée plilosophique qui s'aveugle clle-mêne en tenant pour acquise la réalité d'un sujet transcendantal ne partageant aucunc caractéristique commune aver le monde dans tequel il existe et affichant des qualités qui tendent à faire croire en un sujet désincarné, atemporel, fléteminant. C'est dire que le a caractère historial "du Dasein ne doit pas être retenu d'emblée conme la preuve d'un intérêt particulier que porterait Heidegger à l'histoire, pas plus qu'on ne doit le comprendre comme la seule illustration, ou la meilleure, de l'objectif qui viserait à contrer d'une manière radicale tous les systèmes philosophiques à tendance idéaliste. Car si Heidegger reproche bien à de telles pensées l'ignorance dans laquelle elles se tiennent quant aux déterminités qui * corrompent * l'idéalité du sujet, ce qu'il vise, dans ce reproche, n'est pas de faire 
recomnaître tel ou tel contexte historique particulier et cléterminant, mais bien le principe même d'une telle détermination. Et ce principe ressortit à la rlualité fondatrice de l'être et du temps, dualité qui fonde le Dasein -.. seut élant pour qui la question de l'être se pose - comme constitutivement a jeté ", c'est-à-dire exposé au monde selon des modes qui consacrent sa non-coücidence avec lui-même (donc, sa " non-icléalité " en tant que " sujet"). Pas plus que la " temporalité " du Dasein, son "historicité " ne veut souligner la réalité d'une u histoire " cui suppléerail à la sortie définitive du " paradis " idéaliste et qui ne serait alors qu'un nouvel icléalisne, mais en marche. Co n'est pas l'histoire qui intéresse le Heiclegger de $S Z$; c'est le lemps. Ine: relle confusion qu'engender il est vai presque naturellement Ieidegger, à forede de laiter d'historicilé el rle temporalité - n’est pas absente de l'analyse de Barash. Persuadé de l'imporlance qu'accorde le philosophe à l'u histoire ", il cherehe à mettre à jour la conception que celui-ci s'en laisail, qu'il s'agisse du premice lleidegger, "ontologien-existentialiste n, ou du second, oceupé à la pensée de l'k̂tre. $\hat{\lambda}$ la fin cependant, il convient lui-même que Iteidegger n'est jamais parvenu à exprimer ce concept de l'histoire: "C'est pourguoi l'on peut se demander si la pensée de l'historicité de l'Être, après avoir renoncé à l'analyse ontologique de la linitude de Sein und Zeit. parvient mieux que cette dernière à rendre compte de la plénitude d'un monde historique commun "(p. 187). Mais s'il n'y est jamais parvenu, c'est peut-être notamment parce que cela n'a jamais composé l'essentiel de son projet.

Cela dit, on aurait tort de croire pour autant à une méprise rédhibitoire de la part de Barash. Car non seulement l'œuvre de Heidegger - et ce, malgré que ce clernier affirme ne s'occuper que de problèmes "fondamentaux" ... n'est bicn entendu pas exempte de tout perspectivisme historicue mais, de surcroît, elle comporte bel el bien une réflexion stimulante sur nombre de thèmes philosophicques que l'on aime associcer à des courants ou à des époques particulières (qu'il suffise de nommer ici le néokantisme, la mouvance diltheyenue ou la phénonénologié, lout autant qu'une définition assez unique de l'histoire qu'il convient certainenent d'analyser, Ét l'auteur a par ailleurs tout à fait raison d'affimer qu'une certaine lecture de Heidegger a entièrement oblitéré ces dimensions de son ceuvre. À ce titre, Barash (avec d'autres, tel T. Kisiel) fait office de pionnier pour ce qui est de redonner Heidegger à l'histoire de la philosophie, le "redonner " à cette histoire puisque, auparavant, on se contentait trop souvent soit de penser comme lui (et moins bien), soit de l'ignorer complètenent.

Le premier article du recueil, a Existence el hisloire : la eritique heideggerienne de Karl Jaspers des années 1919-1921 ", a été écrit en 1982 mais revu et augmenté pour la présente édition. Barash y trace le portrait d'un jeune lleidegger persuadé de la nécessité d'une "pensée de l'essentiel ", après que la Prenière Ginerre mondiale ait entrainé la perte de erédibilité autant du néokantisme que de la phénoménologie uaissante. Cette déroute est celle de l'Al/gemeingültigheit, des eritères de validité universelle qu'ont cherchés tout autant les néokantiens que les phénoménologues. En face de celte haute quête se dressent l'existence of la vie, à quoi ees systènes n'onl toujours pas rendu justice. Si le jeune Heidegger partage cette doléance avee Jaspers, il reproche à ce dernier de continuer à miser sur une recherche théorique qui, justement, doit être " détruite "pour que la primauté de l'existence puisse être correctement énoncée. C'est donc longtemps avant $S Z$ que Heidegger s'avise du problème cardinal que représente l'existence de "préconceptions " qui président à la recherche théorique, préconceptions qui doivent elles-mêmes receler quelque caractère plus fondamental que la théorie elle-nême.

" Sur le lieu historique de la vérité : les enjeux herméneutiques chez W. Dilthey et M. Heidegger " date de 1989. Bien que l'intérêt de l'analyse de Barash concernant le dialogue qu'amorce Heidegger avec la réplique au néokantisme que représente l'œuvre de Dilthey soit indéniable, l'auteur place l'ensemble de celle-ci dans une perspective 
selon laquelle l'objectif de Heidegger, dans ce dialogue, serait de "réinterpréter la tâche de la réflexion historique à partir de l'analyse du Dasein "(p. 35). L'article se termine par une conclusion laconique de Barash selon laquelle le problème de la reconstruction "exacte " du passé ne bénéficie pas d'un examen suffisant de la part de Heirlegger. L'absence d'un tel examen, chez Heidegger, étonne d'autant moins si l'on se persuade que le problème de la "réinterprétation de la tâche historique " en est un qui est loujours déjà résolu chè lui (au nom du recouvrement antique de la vérité dont toute la pensée occidentale n'est qu'une conséquencel et qui, done, ne l'intéresse que faiblement. Aussi Barash n'interroge-t-il que sa propre mise en place lorsqu'il écrit : Malgré l'ónome impact souvent tacite de cette tentative (la réinterprétation de la tâche de la réflexion historiquel, Heidegger n'a ensuite plus émis d'opinion sur le mapport entre sa conception de l'histoire exposie dans Scin und Zcit et celle de ses prérlécesseurs qu'il avail lui-même critiqués " (p. 35).

Le troisiène article s'intitule "L'exposition du monkle publie comne problème politique : au sujet de l'interprétation de lleidegger par Hamah Arendt " 1989 . On comnaît l'une des lisèses principales de l'ouvre d'Arendt et gui consiste à transformer Heidegger en arroseur arrosé: $s^{\prime}$ il a cru que toute la pensée occiclentale avant lui restait dominée sans le savoir par un recouvrement inaugural, il ne s'est pas avisé de ce que toule sa pensée à lui el aver elle, toute la pensé oceidentale avant hii ćlait sons le joug d'un mépris fondationnel de la polis, du monde public. Cette interprétation d'Arendt lui permet de conprendre - mais paradoxalement à la lumière de l'analyse heideggerienne du " nonde"-- ce qui a conduit Heidegger à dévaloriser le monde publir: -.. le "On" al à en arriver à proposer lui aussi, sous la forme de son "Analytique existentiale ", une épistémè qui ne se distingue pas fondamentalement de toutes celles qui l'ont précédée mais qui, plutôt, montre comme elles un même rejet méprisant de la doxa. Si Barash expose ici d'une manière claire ce dialogue particulier entre Heidegger et Arendt, curieusement, il ne prend pas lui-même parti. Or, ce dialogue est fondamental pour sa propre thèse, dès lors que si Arendt a raison de reprocher à Heidegger la volonté d'en rester à la quête de vérités éternelles au détriment d'une "vérité " davantage à comprendre comme ce qui doit émaner de l'espace public, Barash a, quant à lui, tort de croire à une explicitation générale qui accorderait au contexte historique ou à l'histoire en général un rôle déterminant pour cette mêne vérité.

"Les seienees de lhistoire el le probleme de la hónogie : le cours de Nartin Heidegger sur " Sam Augustin al le néplatonisme" "1988) s'inserit sous la gouverne générale du problème qui, dans l'Nllemagne du débu du siècle, a opposé la

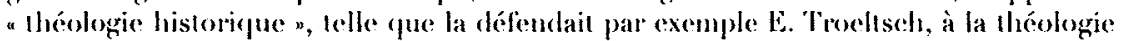
" dialectique" que lui opposait R. Bulmann, ee dernier s'appuyant sur certains éléments de la pensée de Heidegger. Dans la perspentive qui incéresse plus particulièrement Barash, ce dernier comprend l'interprélation de l'histoire qui découle de la pensée d'Augustin iaver, notamment, l'éthique qu'elle impliquej en opposition à celle que fleidegger fait remonter à saint Paul, ainsi qu'à l'exégèse qu'en propose Luther. Cherchant quelle conception de l'histoire cette opposition peut permettre à Heidegger d'obtenir, Barash écrit : si l'on peut done, avec Heidegger, parter d'une. " Listoricité du vrai ", ce ne saurait plus etre en termes de point d'ancrage objectif, mais bien plutôt d'une "répétition", fidèle à l'expérience factice de la vie |... " (p. 83). Ce faisant, il effleure une interpretation valide des eoncepts d'historicité el de temporalité che\% Heidegrer, conecpts qui témoignent bien davantage d'une constitution temporelle et historique de l'existant que d'une intégration de celui-ci dans une histoire pour laquelle il ne devient alors cu'une contingence parmi d'autres. Malheureusement, il n'en tire pas les conclusions nécessaires et continue par la suite de se clemander' si " l'insistance de Heidegger sur le contexte historique, ou encore épochal, cle la 
révélation $\{\ldots \mid$ ne reconduit $|\ldots|$ pas à une pensée de la cohérence objective de l'histoire?" p. 88-89). Bien cư'ume réponse négative soit finalement proposée à cette question, il appert qu'une interprétation juste des comeepts heideggeriens de temporalité et d'historicité laisse peut-être voir d'abord qu'elle ne se pose pas exactement en ces termes.

Dans "Saint Paul, Spinoza et l'absence de l'éthico-politique chez Heidegger" (1993), l'auteur tente de montrer que nême si clle s'en désintéresse, l'ontologie fondamentale de IIcirlegger " porte les Iraces de présupposés nomris par une réflexion sur la théologie n p. 92). Si, à partir de saint Paul, rle buther el, dans une moindre: mesure, d'Augustin, il est intenable de croire en la possibilite de constater l'existence de Dieu grâce à la seule raison, il u'en va pas de mêne pour Thomas d'Aquin et, après lui, pour Spinoza. ()r, selon Barash, on peut etablir une analogie coltre la lantseendance de la foi telle que peurent l'entendere saint Paul ou Luther et la " voix de: la conseience "dont parle $S Z$ : pas plus que la foi no saurait dériver d'une queleonque mélaplussique qui pourtait la préecéder ou la sous-tendre, ne saumait-on déduire une "éthique "à partir d'une réflexion philosophicue. C"est notamment pour he pas se contionter an problène d'un a système o de la pensée qui mène à des considétations chliques ou sociopolitiques que fleidegger a civerait " de proposer une réllexion sur Spinoza.

"Temps de l'être, lemps de l'histoire: Heidegger et son siede " est l'article le plus poussé de ce recueil. L'auteur s'y donne conme tâche de tenter de situer Heidegger clans la perspective du $\mathrm{XX}^{\mathrm{r}}$ siècle. Mieux que tous les autres articles du recucil, celui-ci souligne les problèmes d'une interprétation qui veut situer dans son contexte historique une pensée qui réfute la validité, soire la jossibilité, d'une telle intégration. Bien sûr, entre l'affirmation de Heidegger de ne considérer que des problèmes essentiels qui, fle ce fait, se voient dispensés de nommer le contexte dont ils lirent leur. pertinence, ef l'abstraction réelle rle ceux-ci eu égard à ce contexte, il y a un gouffie, que Heidegger franchit allègrenent mais dont un commentateur se doit de rappeler l'existence. Déjà avertis de ce que la réllexion heideggerieme peut devoir au néokantisne, au " criticisme historique "de Dilthey ou aux spéculations théologicfues, nous sommes ici confiontés au problène du coniexte sociopolitique dans lecpuel se ment l'ouve de Heidegger. Cette confrontation ne se fera pas sans le rappel des sympathies nazies de l'auteur de $S Z$ (dont l'excellent travail biographique récent de R. Safranski dome cependant une plus exacte mesure). Mais examinées dans le contexte général d'une analyse qui vise à éclairer le concept heideggerien d'histoire, ces sympathies cloment, sous la réflexion de Barash, toute la mesure de la difficulté qu'il y a à les bien comprendre, et ce, à l'abri des simplifications parfois étonnantes auxquelles ont souvent donné lieu certains commentaires sur he sujet. Autant le concept heideggerien d'، histoire "fait apparaitre de nombreuses difficultés interprétatives dès lors qu'il concerne d'abord une caractéristique coustitutive du Dasein. bien avant qu'il ne signifie quelque chose pour la compréhension de l'histoire en tant que telle, autant il reste problématique de comprendre en quoi une telle conception de l'histoire entraine, ou n'entraine pas, des conséquences quant aux choix politicjues qui sont ceux de qui la possède. Conscient de cette dualité du problème historique (dualité qui, cependant, est peut-être clavantage le fait de Barash lui-même que de Heidegger'), l'auteur donne ici la mesure de la complexité du rapport qui lie la propre pensée de Heidegger à sa caractérisation du Dasein.

Ce même article présente également une analyse importante de la Kehre, au moins en ce qui a trait aux transformations qui lui sont associées quant au concept heideggerien de !'histoire. Délaissant l'ontologie du Dasein, qui cherche le " sens de l'être ", Heidegger s'est, à un moment (1929-30), tourné plutôt vers la a vérité de l'Être ", qui est aussi son a Histoire ". Pour la première fois alors, le philosophe 
allemand émet nommément quelques détails au sujet de la manière dont il convient de penser l'histoire (en fait, selon les termes de l'"instauration de la vérité "). Malheureusement, ces " détails " révèlent une " histoire "qui est entièrement hors de la portée de la pensée humaine, si ce n'est, pour celle-ci, de la possibilité de "recevoir" quelques éléments qui, notamment par le biais de certaines métamorphoses du langage, peuvent incliquer à l'homme que l'Être se temporalise, s' historise ". Mais quelle pourra bien être, pour la compréhension humaine, une "histoire" dont les déterminations sont incompréhensibles? Il va de soi que Barash ne répondra pas à celte question, pas plus que lleidegger n'v répondait lui-mêne. Nais le commentateur a raison de souligner que, lout aulant que lorsun'il s'agissait de jeter les bases d'une "ontologic foudamentale", s'il s'axil désomais de peuser l'impensable d'une "Histoire de l'Être", dans les deux cas se manifeste la volonté de soustraire l'explication ultime du monde à la pensée rationalisante et théorisante. La primauté de l'existence chaque fois miemne (individualité du Dasein signifie, pour la possibilité d'un système de l'Al/gemein-gr̈higheit, la même chose que la primauté fondationnelle de l'hlistoire de l'Être : une seience, générale et théorique, ne donnera jamais la pleine et entière mesure du réel.

Dans l'article suivant, "L'image du monde à l'époque moderne : les critères de l'interprétation chez le dernier Heiclegger "(1988), Barash examine la manière dont Heidegger peut comprendre les transformations qui président à différentes époques de l'histoire. À l'enseigne de la Scingeschichte, ces transformations sont en fait des métanorphoses de la vétité dont l'unité se fonde dans le moment inaugural du recouvement platonicien. Nais à l'identification, par Hejrlegges, du problématique instant d'une vérité présocratique en guise de fond sur lecquel peuvent être comprises ces métamorphoses, Barash oppose l'influence qu'aurait ene, sur la pensée de ces transformations do la vérité, l'ceuvre hégélienne.

Le demier article du recueil, "La deuxième guerre mondiale dans le nouvement de l'histoire de l'Ête a 1992 , traite une nouvelle fois de l'adhésion de Heidegger au nazisme. L'auteur tente d'identifier une "faille dans l'interprétation heideggerienne de l'histoire " ip. 170, faille qui n'explique peut-être pas entièrement cette adhésion, mais qui doit etre prise en comple pour sa pleine inferprétation. Cette "faille ", Barash l'associe à un irléal d'impartialilé qui préside, ches Hejlegger, au refus de discuter de ses propres partialités (l'auteur cite en eximple l'explical ion que fournit Heidegger à la tragédie de la Seconde Guerre mondiale, explication qui relère d'une fatalité que ne peut expliquer que la Seingeschiche el qui déresponsabilise les acteurs individuels de celte catastrophe a fore de vouloir combaltre le perspectivisme historicue, Heidegger anmil fini par ne plus prendre garde aux conséquences, pour sa propre persée, de sa propre insertion historique. Cóst là un reproche que l'on peut ectainement emvisager d'aclresser à la philosophie de Heirlegger cet que ne manque

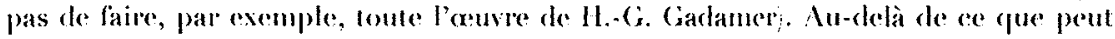
comporter diutile l'examen de ee probleme partieutier, il resterail cependant ici a examiner ee que peut signifier, pour celle question de la déresponsabilisation, l'abandon de la perspective ontologique lelle qu'esquissée par $S Z$ et qui, justement, dévoilait comme l'un de ses monents forts la respensabilisation fondamentale du Dasein chaque fois mien.

Yvon Corbeil

Université de Montréal 